\title{
Valoración del paisaje aplicada a la ingeniería de taludes
}

\author{
José Santiago POzO ANt ONIO \\ Dpto. Ingeniería de los Recursos Naturales y Medio Ambiente \\ Escuela Superior de Minas. Universidad de Vigo
}

Recibido: 14 de Febrero de 2011

Enviado a evaluar: 15 de Febrero de 2011

Aceptado: 5 de Octubre de 2011

\section{RESUMEN}

La necesidad de crear un método que elimine la subjetividad a la hora de valorar un paisaje, es uno de los temas medioambientales en el que en estos últimos años, se ha invertido más tiempo por parte de ambientalistas e ingenieros. Lo que se pretende es extender el concepto tradicional de crear taludes seguros y económicos, a hacer taludes que se integren más en su entorno. Esta técnica puede contribuir a mitigar el impacto paisajístico, haciendo las obras menos nocivas a la vista y a realizar proyectos de explotación con cierres integrados en el entorno. Se hace una comparativa entre un método directo de valoración paisajística y un método indirecto basado en las evaluaciones de una serie de parámetros (fisiografía, elementos artificiales, agua, vegetación, etc.).

Palabras clave: ingeniería de taludes, paisaje, valoración, integración paisajística.

\section{Valuation of landscapes applied to the engineering slopes}

\begin{abstract}
$t$ he need to create a method that eliminates the subjectivity at the moment of valuing a landscape, is one of environmental topics which is more important in the last years. Environmentalists and engineers have been invested a lot of time on this matter .t he purpose that is chased is to extend the traditional concept of creating sure and economic slopes to doing them more integrated in the environment. $t$ his technology can help to mitigate Landscape impact, doing the least harmful works at sight and realizing projects of exploitation with integrated Closings to the environment. It makes a comparative between a direct method of landscape valuation and an indirect method based on the evaluations of a series of parameters (physiography, artificial elements, water, vegetation, etc.).
\end{abstract}

Keywords: engineering slopes, landscape, valuation, landscape integration. 


\section{INTRODUCCIÓN}

El poder ofrecer una opinión sobre la belleza que presenta un paisaje es una tarea que en principio, no tiene por qué suponer dificultades a los observadores, pero en muchas ocasiones la percepción subjetiva puede influir en dicha decisión.

Lo que se pretende es crear una herramienta de trabajo que permita eliminar esos criterios personales de captación y permita una orientación a la hora de evaluar un paisaje. Revisando bibliografía se encuentran sistemas de evaluación referidos al sector turístico, es decir, informan de la potencialidad de dicho paisaje para atraer al visitante, pero lo que se busca en este artículo es el empleo de un método que ayude a los ingenieros ambientales, técnicos o personal cualificado a la hora de tomar decisiones en lo referente a la restauración o rehabilitación de un talud de carretera, una escombrera o una cantera abandonada. Como punto de partida se emplea el trabajo publicado por el Instituto Geológico y Minero de España (IGME): Ordenación Minero-Ambiental del yacimiento de Pizarras Ornamentales de La Cabrera (León) (1995), cuyas directrices se basan en trabajos previos de E. Alberruche, miembro del IGME. Por tratarse de un estudio de detalle, el objetivo es perder la menor cantidad de información posible en el análisis; por lo que se han intentado evaluar todos los aspectos del paisaje que pueden influir en la calidad escénica. Las valoraciones directas por tanto, se han realizado utilizando como fuente de información fotografías. Además para evitar sesgos en la valoración, se emplea un método indirecto que analiza el paisaje a través de descriptores que tendrán en cuenta los diferentes aspectos que influyen en la valoración del paisaje.

Se obtiene, por lo tanto, un método sistemático y objetivo. Para comprobar que los resultados del método son satisfactorios se han comparado los resultados de la valoración por el método indirecto propuesto y la valoración obtenida a través de una encuesta representativa por asignación de valor en escala de 0 a 10. Para llevar a cabo dicha averiguación se dispone de un espacio web www:webs.uvigo.es/alejano donde los participantes pueden completar sus datos personales requeridos y proceder a la valoración de las fotografías. La página web está en español e inglés para acercar esta aplicación al mayor número de personas posible y así facilitar una evaluación paisajística en función de región geográfica, educación, edad y ámbito residencial (urbano o rural).

\section{ESTUDIO DE PAISAJE}

\subsection{DEFINICIÓN}

El paisaje es un concepto que se utiliza de manera diferente en varios campos de estudio, aunque todos los usos del término llevan implícita la existencia de un sujeto observador y de un objeto observado (el terreno) del que se destacan fundamentalmente sus cualidades visuales y espaciales.

Generalmente, el paisaje se define como el resultado de la interacción entre diferentes parámetros físicos y biológicos que gobierna las unidades espaciales de una 
región (Troll, 1971). Otra de las definiciones aportadas por Zonnelveld (1995) es "una parte del espacio sobre la superficie terrestre, la cual consiste en un complejo de sistemas, formado por la actividad de la roca, del agua, del aire, de las plantas, de los animales y del hombre y por su fisonomía, lo que constituye una entidad reconocible". La mayor parte de los estudios de valoración del paisaje, están relacionados con estudios de ordenación del territorio (Villota, 1992). Gómez Orea (1978) justifica la inclusión del paisaje en los estudios de planificación, debido a que se ha convertido en un elemento natural escaso como consecuencia de la presión humana sobre el medio ambiente. En este sentido, Villarino (1985) añade que el paisaje es un recurso difícilmente renovable y fácilmente depreciable. En consecuencia, los propios valores del paisaje se convierten en recurso para el hombre, como elementos de potencial gestión y/o explotación (Leno Cerro, 1993).

Aunque no es fácil definirlo, debido fundamentalmente a la gran cantidad de connotaciones que lleva consigo, el paisaje hace referencia a tres aspectos fundamentalmente (Marí, 2003):

El paisaje como recurso equiparable a los demás recursos naturales.

- El paisaje como elemento de percepción que depende de la experiencia personal, de los conocimientos y de la disposición de cada observador.

- El paisaje entendido como expresión visual y estética del medio, como un concepto integrador que sirve para resumir, desde el punto de vista de la percepción estética, un conjunto de valores ligados a aspectos bióticos y abióticos.

La mayor parte de los estudios de valoración del paisaje están relacionados con estudios de ordenación del territorio, sin embargo, debido a la creciente preocupación por el paisaje y el nuevo marco normativo, se hace necesario la realización de estudios de detalle de los elementos formadores del ambiente (UNESCO, 1972) y hoy en día con las innovaciones de la informática y la sistematización, se plantea de una manera más cómoda y segura (Haines Young, 1993).

Se asume que el suelo y la vegetación son los resultados de la síntesis de parámetros ecológicos y de los elementos del paisaje. Además, el ambiente geomorfológico (determinado por el relieve, el material parental y el tiempo) junto con el clima, son los factores físicos formadores de ese paisaje. La cobertura vegetal y el uso de la tierra constituyen sus factores biológicos y socioeconómicos (Andrade Pérez et al. 1998).

A partir del análisis fisiográfico se desarrolla una clasificación del terreno, la cual tiene un carácter multicategórico que involucra a la mayoría de los elementos medioambientales comprometidos con la génesis de las geoformas y de los suelos que contienen. Esta concepción permite visualizar la relación: clima-paisaje-suelovegetación/uso de la tierra (Villota, 1992).

Se define la unidad de paisaje como un sector básico del territorio que presentan una cierta homogeneidad en sus características perceptuales, así como un cierto grado de autonomía visual (cuenca visual).

El paisaje, entendido como la expresión externa del territorio, es abordado en la Ordenación Minero-Ambiental desde un enfoque estético y perceptual, siendo definido como la percepción polisensorial y subjetiva del medio (González Bernáldez, 1981). 
El paisaje representa un recurso natural y cultural que debe ser considerado en la ordenación y gestión del territorio. Además, dentro del proceso de planificación es contemplado también como un componente del medio, susceptible de ser alterado por la actividad extractiva.

\subsection{METODOLOGÍA DE ESTUDIO DEL PAISAJE}

Para explicar la realidad del paisaje como elemento de síntesis, se han utilizado muchos esquemas, que van desde simples textos descriptivos hasta sofisticados esquemas, con múltiples variables y complejos sistemas de agregación. Según los objetivos del estudio será juicioso elegir un nivel de complejidad adecuado a los plazos y medios disponibles, pero siempre será útil tener en cuenta las variables a utilizar. Esas múltiples variables son de muy diversa procedencia y pueden ser agrupadas de diferentes formas, según la realidad que describen y la intención de su utilización:

- Descriptores físicos: Se refieren a elementos visibles y tangibles del paisaje. Son básicamente cuatro: morfología, vegetación, agua y elementos antrópicos.

- Descriptores artísticos: Se refieren a las dimensiones compositivas formales o abstractas del paisaje, como el color, forma, línea, textura, escala; o también la diversidad.

- Descriptores ecológicos: Se utilizan para explicar el origen y funcionamiento del paisaje, de modo que sus características con reflejo visual no surgen como construcción mental ajena al propio paisaje, sino que vienen explicadas como consecuencia de sus procesos vitales.

- Descriptores psicológicos: Se refieren a los impactos y sensaciones que produce el paisaje en quienes lo observan o experimentan.

Con respecto a las personas encargadas de evaluar los parajes, a través de instantáneas o por visualización directa se debe de considerar que los grupos de población difieren en sus preferencias estéticas frente a un paisaje. Estas diferencias se podrían generar por las distintas experiencias y personalidades de los evaluadores. Pese a esto existen características importantes del paisaje que son universalmente apreciadas. Como se parte del supuesto de que la percepción de un paisaje puede ser diferente según la edad, sexo y actividad profesional, se elige un panel representativo que evaluará las imágenes. Muñoz Pedreros et al. (2000) establecieron la variabilidad derivada de los evaluadores y propusieron técnicas para controlar, dentro de lo posible, esta fuente de variación. En base a ello, se propone un panel de 15 a 20 evaluadores, con una proporción de sexos 1:1 e integrado por tres o cuatro grupos iguales de cinco personas:

- $1^{\text {er }}$ grupo exigente en paisaje: profesionales vinculados al sector del medio ambiente, tales como doctores, ingenieros, etc.

- $2^{\mathrm{o}}$ grupo de transformadores de paisaje: profesionales silvo-agropecuarios, técnicos medio ambientales, etc.

- $3^{\text {er }}$ grupo con adiestramiento en evaluación de paisaje, actuarán como grupo control. 
- $4^{0}$ grupo representativo de las características y localización del paisaje a evaluar: campesinos, tercera edad, etc. Muñoz Pedreros lo considera opcional.

De trabajos anteriores se ha demostrado que el primer grupo son observadores exigentes, por su formación profesional, en cambio los segundos están fuertemente condicionados por el medio construido y son menos exigentes en paisajes naturales (Alejano et al., 2009). Un requisito importante es que los panelistas no conozcan los paisajes a evaluar. Se constató una alta coherencia interna (baja dispersión relativa de las ponderaciones) en los tres grupos estudiados diferenciándose cada grupo (exigentes, transformadores y control), en sus preferencias por los tipos de paisajes estudiados (Muñoz Pedreros et al., 2000).

Como se pretende conformar un panel técnico y normado se deberá escoger evaluadores mayores de edad. Constituido el panel se les explican los objetivos y se les capacita brevemente en la técnica a emplear. Diversos autores han empleado paneles en la misma idea representativa (Paquet et al., 1997) sin embargo no han explorado con paneles de menor tamaño sin perder representatividad.

Craik (1975) desarrolló una lista de 240 adjetivos referentes al paisaje: activo, bello, limpio, cultivado, erosionado, variado, ventoso, etc. A los participantes de la prueba se les pide que seleccionen los adjetivos que consideren descriptivos del paisaje. La elección de los adjetivos ha de ser rápida y después se estudia la frecuencia de asignación de cada adjetivo.

También se ha desarrollado una lista de adjetivos jerarquizados en la idea de Craik (1975) y adaptados por Muñoz Pedreros et al. (1993) a las características culturales y especialmente conceptuales de la población chilena. Los adjetivos están agrupados según la escala universal de Fines (EF), que permitirá asignarle un valor numérico a la valoración nominal.

\section{LEGISLACIÓN}

Este interés por el paisaje ha llevado a la realización de la Convención Europea del Paisaje, cuya resolución fue firmada en Florencia el 20 de octubre de 2000, ratificada por 35 países y que se encuentra en vigor en España desde marzo de 2008 (B.O.E. núm. 31, Martes 5 febrero 2008), por la cual, los estados miembros del Consejo de Europa, mediante diferentes medidas nacionales, transfronterizas y de cooperación europea se comprometen a:

- Reconocer jurídicamente los paisajes.

- Definir y aplicar políticas destinadas a la protección, gestión y ordenación de los mismos.

- Establecer procedimientos para la participación pública.

- Integrar el paisaje en las políticas sectoriales y especialmente en la ordenación territorial y urbanística, para la creación de estructuras estables que se mantengan en el futuro.

Para conseguir lo citado anteriormente, se deben adoptar medidas específicas para así, conseguir los siguientes objetivos: 
- Identificación y valoración de los paisajes.

- La sensibilización y concienciación sobre la conveniencia de su conservación.

- Formación especial en técnicos del paisaje y educación ambiental de los valores del paisaje en la enseñanza, para llegar a un verdadero reconocimiento de su calidad.

Junto a la realización de esta convención, el Año Internacional de las Montañas (2002), supuso la aparición del borrador de la Carta Española de las Montañas (2003), en donde se expone que éstas son una reserva de paisajes que necesita ser conservada, protegida y rehabilitada mediante una serie de objetivos y políticas, que sirvan para el desarrollo económico de estos espacios mejorando la calidad de vida de la población local (Martínez de Pisón, E., 2002).

Sólo así se podrá ir introduciendo en la sociedad y en las actividades económicas, los mecanismos que garanticen el respeto a la calidad del paisaje.

Existe en la sociedad una creencia generalizada de que la extracción y el aprovechamiento de los recursos mineros y la protección del medio ambiente son incompatibles. Esto se debe a que durante mucho tiempo la actividad extractiva se ha desarrollado de una forma desordenada, buscando la máxima rentabilidad económica sin tener en cuenta los costes ambientales que pudieran derivarse. La extracción de los recursos minerales a cielo abierto implica generalmente unos períodos de ocupación de los terrenos, que no superan los 20 o 30 años, salvo casos especiales como los grandes yacimientos metálicos. El abandono de estas áreas debe realizarse de tal forma que los terrenos afectados vuelvan a ser útiles para un determinado uso sin perjudicar el medio ambiente. La rehabilitación o restauración de la zona afectada se entiende como un equilibrio entre desarrollo económico y conservación de la naturaleza. Es una idea universalmente aceptada que la garantía de que el desarrollo de las actividades humanas se produzca en equilibrio con la protección del medio, pasa por una adecuada planificación del territorio (Desarrollo Sostenible). Dicho compromiso sólo puede ser alcanzado en el marco de una adecuada Ordenación Territorial, que garantice el uso racional del suelo y de sus recursos. El objetivo último de la planificación territorial es la distribución espacial de las actividades, dentro de un marco estratégico de crecimiento definido, de forma que se garantice el desarrollo económico equilibrado, la mejora de la calidad de vida, la gestión responsable de los recursos naturales y la protección ambiental, y el uso racional del territorio (Carta Europea O.T., 1983). Esta distribución de usos, entre ellos, el minero, debe realizarse de acuerdo con los siguientes principios:

- Adaptación a la capacidad de acogida del territorio.

- Optimización de las interacciones de las actividades a localizar.

- Uso múltiple del territorio (superposición de actividades compatibles en espacio y/o tiempo; aproximando las que son complementarias; y alejando las incompatibles).

Por otra parte, la planificación territorial ejerce un papel integrador de las políticas sectoriales, y es desde esta perspectiva global e integradora, como se debe abordar la problemática medioambiental derivada de la actividad extractiva. La impor- 
tancia económica de la explotación de los recursos minerales y su creciente demanda, y la mayor sensibilidad social ante los problemas ambientales, hacen necesario armonizar ambos intereses a fin de garantizar un desarrollo sostenible, y es en este contexto, donde se inscribe la ordenación minero-ambiental.

El Instituto Geológico y Minero de España (IGME) tiene como cometido, entre otras funciones, el estudio, inventario y evaluación de los recursos geológicos y minerales considerados como un recurso no renovable, para propiciar su uso ordenado y compatible con la conservación del medio.

\section{RESTAURACIÓN DE TALUDES}

La construcción de taludes, carreteras y otras obras públicas produce impactos ambientales severos que afectan a las propiedades de los ecosistemas, tales como la fragmentación de hábitats, la alteración de la estructura de los ecosistemas, elevadas tasas de erosión y transporte de sedimentos o compactación del suelo (Forman y Deblinger, 2000). De hecho, en zonas degradadas por construcción de taludes, se han llegado a medir pérdidas por erosión hasta 15 veces mayores que en las zonas agrícolas colindantes (Navarro y Jonte, 1996). Además, las infraestructuras suelen producir un impacto paisajístico y una pérdida de funciones del ecosistema anteriores a la perturbación. Toda esta serie de impactos ambientales hace necesaria la restauración del ecosistema. Ahora bien, para llevar a cabo una restauración correcta es necesario tener un conocimiento previo de la ecología de los ecosistemas a restaurar (Ervin H., 1987). Los taludes generados en estas obras presentan escasas coberturas vegetales y el establecimiento de la vegetación, tanto de forma natural como a través de restauraciones, es muy lento. Al igual que en otros ambientes, en los taludes la colonización se ve limitada por la restringida capacidad de dispersión de algunas semillas, por lo que estas laderas son colonizadas principalmente por especies provenientes de áreas cercanas y/o por especies capaces de dispersarse a larga distancia. Existen una serie de limitaciones edáficas que hacen que, aunque muchas semillas sean capaces de llegar a los taludes, la colonización no se produzca. En general, las propiedades físicas y químicas de las zonas alteradas no son adecuadas para el establecimiento de la vegetación, ya que presentan suelos poco fértiles (escasez de nutrientes y de materia orgánica), de texturas poco equilibradas y/o pedregosos, el factor que más limita y modula la colonización vegetal en taludes de carreteras en ambientes semiáridos es el estrés hídrico. Se emplean tratamientos como el uso de tierra vegetal o la aplicación de mulches que pueden atenuar las condiciones limitantes y aceleran la colonización vegetal. Además, en desmontes, se han obtenido resultados prometedores utilizando tratamientos que disminuyen la compactación, mejoran las condiciones del suelo localmente y permiten la penetración de las raíces (Tormo et al., 2009). El conocimiento de las limitaciones de la colonización vegetal en los distintos tipos de taludes y del beneficio de determinados tratamientos para el establecimiento de las plantas ha de permitir optimizar el uso de los recursos según las necesidades de cada zona a restaurar y de elaborar consecuentemente, planes de revegetación con garantías de éxito. 


\section{METODOLOGÍA DE LA EXPERIENCIA}

Existen tres tipos de métodos para poder valorar la calidad de un paisaje: métodos directos, indirectos y mixtos (Alberruche, 2005). El primero se caracteriza porque la evaluación del paisaje se realiza por medio de la contemplación del mismo, bien en el campo o por medio de algún soporte gráfico. Con respecto a los métodos indirectos, estos forman el grupo más numerosos de técnicas de valoración y son también los más antiguos. Incluyen métodos cualitativos y cuantitativos que evalúan el paisaje analizando y describiendo sus componentes o descriptores. Los mixtos intentan combinar las ventajas inherentes a los métodos directos e indirectos. Se basa en la idea de que la valoración sólo se puede realizar de forma directa, pero utilizan la desagregación en componentes para contrastar la valoración directa o como instrumento para facilitar o simplificar dicha valoración. Además proporciona datos relevantes acerca de las actuaciones sobre el territorio. El procedimiento con dicho análisis está constituido por los siguientes pasos:

- Se realiza una valoración directa subjetiva adjudicando un valor.

- Se selecciona una serie de componentes del paisaje y se valoran.

- Se efectúa un análisis mediante correlación lineal, para calcular la importancia relativa o ponderación de cada variable. De esta manera se eliminan los componentes no significativos y a continuación, se recalculan los pesos de las variables finales.

- Se realiza una valoración completa del territorio.

Se toma como base, el esquema de valoración aplicado en el proyecto de ordenación minero-ambiental del yacimiento de pizarras ornamentales de La Cabrera (León) (ITGE, 1995; Alberruche, 2002) para la evaluación de la calidad visual del paisaje, conocido como valor paisajístico (VPAI), cuyo esquema se presenta en la Tabla 1.

En el esquema de valoración propuesto se aplica el método de desagregación en componentes (Gómez Orea, 1994) en paisaje intrínseco y extrínseco.

$$
V P A I=0,75 * P I N+0,25 * P E X
$$

donde PIN=paisaje intrínseco; PEX=paisaje extrínseco.

El paisaje intrínseco (PIN) se define como la percepción que de una unidad territorial o paisajística, obtiene un observador situado en cualquier punto del entorno desde el que dicha unidad, es accesible a la percepción polisensorial (Alejano et. al., 2009). Equivale, por lo tanto, a una consideración de la unidad como emisora de vistas:

$$
P I N=0,1 * F I+0,1 * A G+0,2 * V+0,2 * E A+0,4 * C M
$$

donde $\mathrm{FI}=$ fisiografía o morfología; $\mathrm{AG}=$ agua; $\mathrm{VG}=$ vegetación; $\mathrm{EA}=$ elementos artificiales; $\mathrm{CM}=$ composición.

El paisaje extrínseco se define como la precepción que obtiene un observador situado en una determinada unidad paisajística (unidad territorial) del entorno, que le rodea (puede abarcar una o varias unidades territoriales). Este concepto recoge el Potencial de Vistas de cada una de las unidades territoriales.

$$
P E X=0,4 * P R+0,4 * C T+0,2 * P O
$$


donde $\mathrm{PR}=$ profundidad del campo de visión; $\mathrm{CT}=$ calidad del tema de las vistas; $\mathrm{PO}=$ posición altitudinal.

Tabla 1. Esquema del método VPAI

\begin{tabular}{|l|}
\hline \multicolumn{1}{|c|}{ PAI= 0,75. PIN + 0,25.PEX } \\
\hline \multicolumn{1}{|c|}{ PIN = 0,1.FI+0,1.AG+0,2.VG+0,2.EA+0,4.CM } \\
PIN: PAISAJE INTRÍNSECO \\
AG: Fisiografía \\
$\begin{array}{l}\text { VG: Vegetación } \\
\text { EA: Elementos artificiales } \\
\text { CM: Composición }\end{array}$ \\
\hline \\
$\begin{array}{l}\text { PR: Profundidad de visión } \\
\text { CT: Calidad de tema } \\
\text { PO: Posición altitudinal }\end{array}$ \\
\hline
\end{tabular}

Fuente: Alberruche, 2005

\subsection{PAISAJE INTRÍNSECO (PIN)}

\subsubsection{DESCRIPTORES FÍSICOS}

\subsubsection{Fisiografía o morfología (fi)}

Este factor describe las características fisiográficas o geomorfológicas dominantes en cada unidad del paisaje. En este descriptor están incluidos los componentes de la forma del paisaje, como pueden ser la pendiente (P), el desarrollo vertical (DV) y los rasgos especiales o característicos que pueda tener la topografía (F). En este caso se han considerado 2 modelos de valoración, que se han ponderado al $50 \%$ cada uno:

$$
F I=\frac{S M+D V}{2}
$$

donde $\mathrm{SM}=$ singularidad morfológica; $\mathrm{DV}=$ desarrollo vertical.

La variable SM es la suma de los factores pendiente $(\mathrm{P})$ y formas morfológicas (F), los cuales están basados en la calidad escénica de los rasgos biofísicos (USDA Forest Service, 1974). Ver Tablas 2 y 3.

$$
S M=P+F
$$

donde $\mathrm{P}=$ pendiente; $\mathrm{F}=$ Formas morfológicas.

La variable DV valora el desarrollo vertical y la complejidad topográfica. Divide la topografía en cuatro clases, siendo agrupadas en un mismo grupo las clases accidentado y montañoso, como se ve en la Tabla 4. 
Tabla 2. Valoración de la pendiente

\begin{tabular}{|l|c|}
\hline \multicolumn{1}{|c|}{ Pendiente } & Valor $\mathbf{P}$ \\
\hline Pendiente $>\mathbf{6 0 \%}$ & 3 \\
\hline Pendiente $\mathbf{3 0 - 6 0 \%}$ & 2 \\
\hline Pendiente 3-30\% & 1 \\
\hline Sin pendiente $\mathbf{0 - 3 \%}$ & 0 \\
\hline
\end{tabular}

Fuente: USDA Forest Service, 1974

Tabla 3. Valoración de las formas morfológicas.

\begin{tabular}{|l|c|}
\hline \multicolumn{1}{|c|}{ Formas morfológicas } & Valor F \\
\hline $\begin{array}{l}\text { Formas rocosas sobresalientes } \\
\text { Pedrizas, afloramientos y taludes inusuales en tamaño, forma y localización }\end{array}$ & 2 \\
\hline Rasgos obvios pero que no resaltan & $\mathbf{1}$ \\
\hline Sin rasgos apreciables & $\mathbf{0}$ \\
\hline
\end{tabular}

Fuente: USDA Forest Service, 1974.

Tabla 4. Valoración del desarrollo vertical

\begin{tabular}{|l|l|c|}
\hline \multicolumn{1}{|c|}{ Desarrollo vertical } & \multicolumn{1}{c|}{ Diferencias de altitud en $\mathbf{4} \mathbf{~ k m} \mathbf{2}$} & Valor DV \\
\hline Accidentado-montañosos & $>150 \mathrm{~m}$ & 5 \\
\hline Ondulado & $30-150 \mathrm{~m}$ & 4 \\
\hline Llano & $<30 \mathrm{~m}$ & 1 \\
\hline
\end{tabular}

Fuente: Alejano et al., 2009

\subsubsection{Agua (AG)}

Se valora la presencia o ausencia de agua, y las formas en que ésta se manifiesta en el territorio. En las siguientes tablas (Tablas 5, 6 y 7) se presentan los parámetros y las valoraciones a considerar al realizar la evaluación (USDA, 1974; BLM, 1980). Se clasifica en primer lugar la importancia del elemento acuoso a valorar (lagos, mares, ríos, nieve o hielo). En segundo lugar se tienen los factores singulares susceptibles de generar escenarios atractivos para el observador. Estos factores son sumados a la valoración del tamaño de los lagos y mares, de los ríos y de la superficie de la nieve. 
Tabla 5. Valoración de lagos y mares

\begin{tabular}{|l|c|}
\hline \multicolumn{1}{|c|}{ Tamaños lagos y mares } & Valor TLM \\
\hline Mar, grandes lagos & 3 \\
\hline Lagos medianos & 2 \\
\hline Lagos pequeños & Valor SLM \\
\hline Factores que hacen variar la calidad escénica & De 1 a 3 \\
\hline $\begin{array}{l}\text { Reflejos abundantes y formas de bordes irre- } \\
\text { gulares } \\
\text { Se forman olas } \\
\begin{array}{l}\text { Islas, deltas } \\
\text { Acantilados, playas } \\
\text { Estuarios }\end{array}\end{array}$ \\
\hline $\begin{array}{l}\text { Malos olores } \\
\text { Aguas sucias }\end{array}$ & De -1 a -5 \\
\hline
\end{tabular}

Fuente: Alejano et al., 2009

Finalmente para valorar el descriptor se escoge el valor más alto de TLM+SLM, $\mathrm{TR}+\mathrm{SR}, \mathrm{TN}+\mathrm{SN}$.

Tabla 6. Valoración de ríos y corrientes de agua

\begin{tabular}{|l|c|}
\hline \multicolumn{1}{|c|}{ Tamaños de los ríos } & Valor TR \\
\hline Grandes ríos & 3 \\
\hline Cursos de agua comunes en recorrido y caudal & 2 \\
\hline Arroyos intermitentes & Valor SR \\
\hline Factores que hacen variar la calidad escénica & De 1 a 3 \\
\hline $\begin{array}{l}\text { Meandros cambios de cauce } \\
\text { Rápidos y cascadas } \\
\text { Deltas } \\
\text { Estuarios }\end{array}$ & De -1 a -5 \\
\hline $\begin{array}{l}\text { Malos olores } \\
\text { Aguas sucias }\end{array}$ & \\
\hline
\end{tabular}

Fuente: Alejano et al., 2009

Tabla 7. Valoración del afecto de nieve o hielo

\begin{tabular}{|l|c|}
\hline \multicolumn{1}{|c|}{ Superficie de hielo o nieve } & Valor TN \\
\hline Manto continuo de nieve & 3 \\
\hline Zonas de nieve abundante & 2 \\
\hline Restos de nieve o de helada en las zonas más frías & 1 \\
\hline Factores que varían la calidad escénica & Valor SN \\
\hline $\begin{array}{l}\text { Estalactitas de hielo } \\
\text { Nieve en los árboles } \\
\text { Grandes acumulaciones de nieve o hielo (espesor) }\end{array}$ & De 1 a 3 \\
\hline
\end{tabular}

Fuente: Alejano et al., 2009 


\subsubsection{Vegetación (VG)}

Se valora la relevancia paisajística que determinadas formaciones vegetales adquieren espacialmente. Se han empleado tres descriptores:

Tipo de vegetación: clímax (formaciones boscosas), prados y bosques ribereños, monte bajo, tierras de cultivos, vegetación rupícola y ausencia de vegetación (IGME, 1995; Alberruche, 2002).

- Densidad vegetativa (Muñoz Pedreros, 2004).

- Diversidad de vegetación (USDA Forest Service, 1974; BLM, 1980).

- Los valores asignados a cada parámetro están reflejados en las Tablas 8, 9 y 10.

$$
V G=\frac{\text { Tipo de cubierta }+ \text { Diversidad }+ \text { Densidad }}{2}
$$

Tabla 8. Valoración del tipo de cubierta vegetal

\begin{tabular}{|l|c|}
\hline \multicolumn{1}{|c|}{ Tipo de cubierta vegetal } & Valor \\
\hline Vegetación clímax & 5 \\
\hline Mosaico de prados y bosque ribereño & 4 \\
\hline Matorral, repoblaciones, monte bajo y pastizal & 3 \\
\hline Pastizales y tierras de cultivo & 2 \\
\hline Vegetación rupícola & 1 \\
\hline Sin vegetación & 0 \\
\hline
\end{tabular}

Fuente: IGME, 1995 y Alberruche, 2005

La vegetación al igual que el agua es un factor que añade calidad visual al paisaje, caracterizándolo y proporcionando una cubierta natural al suelo. Por tanto, la superficie ocupada por la vegetación es un factor importante a tener en cuenta. Como las valoraciones se realizan a través de fotografías, se considerará el porcentaje ocupado por vegetación en la totalidad de la instantánea.

Tabla 9. Valoración de la diversidad de especies vegetales

\begin{tabular}{|l|c|}
\hline \multicolumn{1}{|c|}{ Diversidad de especies vegetales } & Valor \\
\hline Alto grado de diversidad de especies, con formas, texturas y distribución interesantes & 5 \\
\hline Diversidad media & 3 \\
\hline Monocultivo & 1 \\
\hline Sin vegetación & 0 \\
\hline
\end{tabular}

Fuente: Alejano et al., 2009 
Tabla 10. Valoración de la superficie ocupada por la vegetación

\begin{tabular}{|l|c|}
\hline \multicolumn{1}{|c|}{ Área ocupada por la vegetación } & Valor \\
\hline $\mathbf{8 0 - 1 0 0 \%}$ área cubierta & 5 \\
\hline $\mathbf{6 0 - 8 0} \%$ área cubierta & 4 \\
\hline $\mathbf{4 0 - 6 0 \%}$ área cubierta & 3 \\
\hline $\mathbf{2 0 - 4 0 \%}$ área cubierta & 2 \\
\hline $\mathbf{1 - 2 0} \%$ área cubierta & 1 \\
\hline Sin vegetación & 0 \\
\hline
\end{tabular}

Fuente: Alejano et al., 2009.vegetación

\subsubsection{Elementos artificiales (EA)}

Esta variable define el efecto de la actividad humana sobre el medio, a través del grado de integración o discordancia de los distintos usos en el territorio. En ella analizamos en qué medida la calidad de un paisaje puede ser enriquecida, o por el contrario, empeorada a tenor de la receptividad escénica. Entre los elementos que pueden añadir calidad al paisaje están los elementos singulares de carácter monumental y entre los que la restan se pueden incluir canteras, industrias, plantas de tratamiento, líneas de electricidad, ferrocarriles, carreteras, edificaciones, etc.

Se han evaluado a través de la sensación provocada por el paisaje sobre el observador (Se) y la escala de alteración o magnitud del impacto producido (Al). La escala de alteración se considera un factor secundario que tiene poca importancia cuando la sensación es buena, y mucha cuando el impacto del elemento artificial es negativo, ya que es un indicativo de la magnitud del impacto producido. Se emplea la siguiente fórmula (Ec. 8) en la que el peso de alteración varía entre 0,6 y 0,1 según la sensación sea muy desagradable o se trate de elementos singulares (Alejano et al., 2009).

$$
E A=S e *(0,4+0,1 * S e)+A l *(0,6-0,1 * S e)
$$

donde $\mathrm{Se}=$ sensación provocada por la instantánea; $\mathrm{Al}=$ escala de alteración.

La sensación ( $\mathrm{Se}$ ) es una variable con cierta subjetividad, ya que hay que tener en cuenta no sólo aspectos visuales sino todas las sensaciones percibidas debidas únicamente al elemento artificial, por ejemplo, malos olores por basureros, polvo derivado de una planta de tratamiento de áridos, etc. Estos aspectos afectan sin duda a la calidad del paisaje, ya que forman parte de la percepción del observador. La tabla empleada en el método (Tabla 11) está basada en los adjetivos jerarquizados traducidos al castellano por Muñoz Pedreros et al. (2004) en la idea de Craik (1975), comentada en el apartado 2.2. Se ha reducido la cantidad de adjetivos, pues está diseñado para valorar una parte del paisaje, y no para el paisaje en su totalidad. Se intentan resaltar zonas poco atractivas para el observador, como pueden ser los taludes de cantera, con el objetivo de tener un instrumento de apoyo para realizar un mejor diseño de los mismos, a través de las pertinentes medidas correctoras. Entre los adjetivos seleccionados se dispone de los relacionados con las siguientes temáticas:

- Aquellos que pudieran ser calificados como de interés turístico. 
- La ausencia de elementos artificiales se considera también positivamente, por lo tanto tendrá también, la máxima puntuación. Este no va a ser el objetivo de nuestros estudios, sin embargo, debemos hacer notar que una buena restauración puede hacer que su existencia no se considere negativa para el paisaje, sino que se encuentre integrada en el entorno o incluso constituya un elemento que aporte diversidad al paisaje, sin producir una alteración negativa.

Es destacable para este estudio, que los taludes de cantera desprovistos de vegetación, habitualmente suelen reducir la calidad visual, por lo cual se ha considerado que son feos, y se le ha otorgado un valor de 1, el cual se recomienda para los taludes típicos. Naturalmente pueden encontrarse taludes especialmente bonitos o muy desagradables a los que subjetivamente y a criterio del observador podrían otorgársele otras valoraciones.

La escala de alteración (Al) da una idea de la magnitud de la alteración producida y está basado en descriptores artísticos. Para calcularlo se emplea la siguiente ecuación:

$$
A l=\frac{M l+F}{2}
$$

donde: $\mathrm{Ml}=$ modificación de las líneas del paisaje; $\mathrm{F}=$ configuración espacial.

La configuración espacial es un elemento visual complejo, que engloba el conjunto de cualidades del paisaje, determinadas por la organización tridimensional de los objetos y los espacios libres o vacíos de la escena, como se puede observar en la Tabla 13. La configuración espacial de los componentes que integran la escena define distintos tipos de paisaje:

- Panorámicos: no existen límites aparentes para la visión y predomina lo horizontal.

- Cerrados: definidos por la presencia de barreras visuales que determinan una marcada definición del espacio.

- Focalizados: Caracterizados por la existencia de líneas paralelas u objetos alineados que parecen converger hacia un punto focal que domina la escena.

- Dominados por un elemento singular, una catarata, una forma prominente del terreno, un árbol aislado, etc.

- Filtrados por la presencia de una pantalla arbórea abierta que permite el paso de la visión a través de ella.

La organización espacial de la escena se traduce en una estructura visual o composición de las partes diferenciadas según una distribución determinada. En ella se distinguen unas pautas de color, de forma, de líneas y de textura que tendrán una cierta escala y se distribuirán espacialmente de una forma determinada. Dentro del espacio visual definido tiene importancia la posición espacial de los componentes del paisaje, determinada por su posición topográfica (Alejano et al., 2009): en llano, en fondo de valle, a media ladera, en el borde superior de la ladera o en la cresta.

Así como el fondo escénico contra el que se recortan: el cielo, el agua, el terreno, la vegetación o las construcciones. 
Las posiciones elevadas y expuestas tienden a resaltar el objeto, mientras que las bajas y protegidas tienden a ocultarlo. De la misma forma, un objeto visto contra el cielo o el agua destaca más, por lo general, que cuando se ve contra el terreno.

Tabla 11. Valoración de la sensación provocada

\begin{tabular}{|l|c|}
\hline \multicolumn{1}{|c|}{ Sensación provocada por el elemento artificial } & Valor Se \\
\hline Elementos singulares o ausencia de elementos & 5 \\
\hline Interesante & 4 \\
\hline Agradable & 3 \\
\hline Sin interés & 2 \\
\hline Feo & 1 \\
\hline Muy desagradable & 0 \\
\hline
\end{tabular}

Fuente: Alejano et al., 2009

Tabla 12. Valoración de la modificación de las líneas del paisaje

\begin{tabular}{|l|c|}
\hline \multicolumn{1}{|c|}{ Modificación de las líneprincipales del paisaje } & Valor MI \\
\hline No modifica las líneas del paisaje & 5 \\
\hline Modificación leve de las líneas del paisaje & 4 \\
\hline Modificación moderada de las líneas del paisaje & 3 \\
\hline Modificación importante de las líneas del paisaje & 2 \\
\hline Modificación importante de la línea de horizonte & 1 \\
\hline Modificación global del paisaje & 0 \\
\hline
\end{tabular}

Fuente: Alejano et al., 2009

Tabla 13. Valoración de la configuración espacial

\begin{tabular}{|l|c|}
\hline \multicolumn{1}{|c|}{ Grado inancia del elemento artificial } & Valor F \\
\hline Pasa desapercibido & 5 \\
\hline Elemento no dominante & 3 \\
\hline Elemento dominante o focalizado & 1 \\
\hline Afecta al conjunto del paisaje & 0 \\
\hline
\end{tabular}

Fuente: Alejano et al., 2009

\subsubsection{DESCRIPTORES ARTÍSTICOS}

\subsubsection{Composición (CM)}

La composición se define como un componente de síntesis, resultado de la combinación de los distintos elementos visuales que conforman el medio físico, biótico y humano.

$$
C M=0,5 * i+0,5 * c
$$

donde i=interacción, valorada en función de la complejidad o diversidad y la armonía o naturalidad; $\mathrm{c}=$ cromatismo, valorado descomponiéndolo en tinte, tono, brillo y contraste. 
La interacción define el grado de complejidad o número de elementos que se combinan, y el grado de armonía o naturalidad en el que los diferentes componentes del paisaje se han combinado.

$$
i=0,3 * c p+0,7 * a r
$$

donde $\mathrm{cp}=$ complejidad; ar=armonía.

La complejidad (cp) valora la diversidad del pasaje, que es considerada como un factor positivo dentro del mismo, los paisajes monótonos son menos atractivos para el espectador (Tabla 17).

La armonía o naturalidad (ar) se define como el porcentaje de superficie que estos elementos ocupan en el conjunto de la superficie total de la unidad y sus valoraciones se presentan en la Tabla 18 .

Con respecto al cromatismo se valora el conjunto de la composición paisajística en función de criterios de diversidad, variabilidad estacional y contraste cromático. Se valora en función de sus cuatro características más destacadas:

$$
\text { CROMATISMO }=\frac{\text { Tinte }+ \text { Tono }+ \text { Brillo }+ \text { Contraste }}{\mathbf{4}}
$$

En el caso del Tinte, los valores cálidos (rojizos o amarillos) tienen mayor valoración, al contrario que los fríos como los verdes y azules (Tabla 14). Igualmente ocurre con el tono ya que los colores claros tienen mayor nota que los oscuros (Tabla 15). Para valorar los brillos, los colores brillantes tienen nota más alta y los mates más baja (Tabla 16).

Tabla 14. Valoración del tinte

\begin{tabular}{|l|c|}
\hline \multicolumn{1}{|c|}{ Valoración del tinte } & Valor \\
\hline Colores cálidos & 5 \\
\hline Colores predominantemente cálidos & 4 \\
\hline Alternancia de colores cálidos y fríos & 3 \\
\hline Colores predominantemente fríos & 2 \\
\hline Colores fríos & 1 \\
\hline
\end{tabular}

Fuente: Alejano et al., 2009

Figura 2. Clasificación del tinte

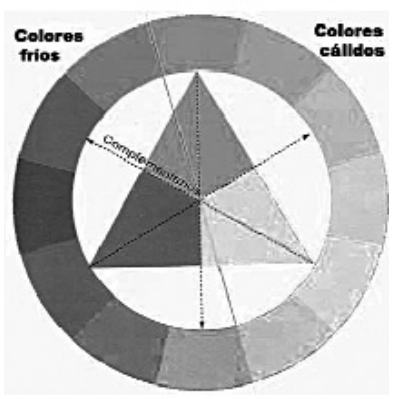

Fuente: Propia 
Tabla 15. Valoración del tono

\begin{tabular}{|l|c|}
\hline \multicolumn{1}{|c|}{ Valoración del tono } & Valor \\
\hline Colores claros & 5 \\
\hline Colores predominantemente claros & 4 \\
\hline Alternancia de colores claros y oscuros & 3 \\
\hline Colores predominantemente oscuros & 2 \\
\hline Colores oscuros & 1 \\
\hline
\end{tabular}

Fuente: Alejano et al., 2009

Tabla 16. Valoración del brillo

\begin{tabular}{|l|c|}
\hline \multicolumn{1}{|c|}{ Valoración del brillo } & Valor \\
\hline Colores brillantes & 5 \\
\hline Colores predominantemente brillantes y mates & 4 \\
\hline Alternancia de colores brillantes y mates & 3 \\
\hline Colores predominantemente mates & 2 \\
\hline Colores mates & 1 \\
\hline
\end{tabular}

Fuente: Alejano et al., 2009

Tabla 17. Valoración de la fragmentación

\begin{tabular}{|l|c|c|}
\hline \multicolumn{1}{|c|}{ Clasificación de la fragmentación } & Manchas/km2 & Valor cp \\
\hline Muy alta & $>8$ & 5 \\
\hline Alta & $6-7$ & 4 \\
\hline Media & $5-6$ & 3 \\
\hline Baja & $3-4$ & 2 \\
\hline Muy baja & $2-3$ & 1 \\
\hline
\end{tabular}

Fuente: Alejano et al., 2009

Tabla 18. Valoración de la armonía o naturalidad

\begin{tabular}{|l|c|}
\hline \multicolumn{1}{|c|}{$\begin{array}{c}\text { Superficie total-\% } \\
\text { Superficie formada por elementos artificiales }\end{array}$} & Valor ar \\
\hline $\mathbf{8 0 0 \%}$ & 5 \\
\hline $\mathbf{6 0 - 8 0} \%$ & 4 \\
\hline $\mathbf{4 0 - 6 0 \%}$ & 3 \\
\hline $\mathbf{2 0 - 4 0 \%}$ & 2 \\
\hline $\mathbf{1 - 2 0 \%}$ & 1 \\
\hline $\mathbf{0 \%}$ & 0 \\
\hline
\end{tabular}

Fuente: Alejano et al., 2009

\subsection{PAISAJE EXTRÍNSECO (PEX)}

\subsubsection{AMPLITUD Y PROFUNDIDAD DEL CAMPO DE VISIÓN (PR)}

La valoración de este descriptor se realiza teniendo en cuenta los siguientes criterios (Alberruche, 2005):

- Profundidad de visión lejana, que se correspondería con el plano poco visible (B.L.M., 1980), y se le adjudica la distancia del límite extraocular, es decir, mayor de $3 \mathrm{~km}$. 
- Profundidad de visión a media o alta distancia, que se correspondería con el plano de fondo, cuya distancia estaría entre $1500 \mathrm{~m}$ y el límite extraocular.

- Profundidad de visión a media distancia, que se correspondería con el $2^{\circ}$ plano (700-500 m).

- Profundidad de visión próxima, que se correspondería con el $1^{\circ}$ plano, distancia menor de $700 \mathrm{~m}$.

- Profundidad de visión inmediata, que se ha establecido en $50 \mathrm{~m}$.

Las valoraciones en función de las amplitudes del campo de visión se encuentran en la Tabla 19.

Tabla 19. Valoración de la amplitud y profundidad del campo de visión

\begin{tabular}{|l|c|c|}
\hline Clasificación de la amplitud del campo de visión & Distancia del campo de visión & Valor PO \\
\hline Lejanas & $>3000 \mathrm{~m}$ & 5 \\
\hline Media/alta distancia & $1500-3000 \mathrm{~m}$ & 4 \\
\hline A media distancia & $700-1500 \mathrm{~m}$ & 3 \\
\hline Próxima & $200-700 \mathrm{~m}$ & 2 \\
\hline Inmediata & Hasta $50 \mathrm{~m}$ & 1 \\
\hline
\end{tabular}

Fuente: Alejano et al., 2009

\subsubsection{CALIDAD DEL TEMA DE LAS VISTAS (CT)}

Se trata en este caso de evaluar el atractivo que pueda tener una determinada entidad para atraer a posibles observadores (Alberruche, 2005).

Tabla 20. Valoración de la calidad del tema de las vistas

\begin{tabular}{|l|c|}
\hline \multicolumn{1}{|c|}{ Calidad del tema } & Valor CT \\
\hline Excelente & 5 \\
\hline Buena & 4 \\
\hline Regular & 3 \\
\hline Mala & 2 \\
\hline Muy mala & 1 \\
\hline
\end{tabular}

Fuente: Alejano et al., 2009

Un observador se va a sentir muy atraído por el paisaje que le ofrece un parque natural, o una zona monumental, sin embargo se va a sentir mucho menos atraído por el paisaje que le puede ofrecer un poblado de chabolas, un basurero o un polígono industrial. La evaluación es subjetiva.

\subsubsection{POSICIÓN ALTITUDINAL (PO)}

Valora la diferencia de calidad escénica producida por la posición del observador con respecto a la cuenca visual. La altura relativa de observación es la variable que domina la sensación de dominio. Mirar desde arriba hacia abajo produce cuencas visuales amplias y hace visibles las relaciones entre los diferentes componentes del paisaje. Desde la altura, los montes, los valles, los ríos, la costa, y el mar, en su caso, 
son percibidos como producto del relieve y adquieren un sentido visual unitario. De forma recíproca, la visión inferior amplifica la impresión visual de los objetos altos como los edificios. Según Higuchi (1975), con ángulos visuales por encima de $27^{\circ}$ el objeto llena totalmente la cuenca visual y sólo se perciben los detalles. Según las clasificaciones de Alberruche (2005) y las referencias aportadas por Higuchi (1975) se realiza la valoración de este parámetro, como se muestra en la siguiente Tabla.

Tabla 21. Valoración de la posición altitudinal

\begin{tabular}{|l|c|}
\hline \multicolumn{1}{|c|}{ Ángulo de visión sobre la horizontal } & Valor PO \\
\hline $15^{\circ}$ superior & 5 \\
\hline $\mathbf{9}^{\circ}$ superior & 4 \\
\hline $\mathbf{< 3}^{\circ}$ (a nivel) & 3 \\
\hline $\mathbf{9}^{\circ}$ inferior (La montaña es observada como tal) & 2 \\
\hline $\mathbf{1 5}^{\circ}$ inferior (Domina la percepción de ladera) & 1 \\
\hline
\end{tabular}

Fuente: Alejano et al., 2009

\section{ENCUESTA}

Para la realización de la experiencia se emplean como soporte, fotografías, concretamente 33; las cuales tratan de abarcar las distintas puntuaciones posibles y enfocadas hacia la valoración de taludes de carretera, de minas, escombreras, etc. Tras la valoración indirecta se obtienen tablas como la siguiente:

Figura 3. Valoración indirecta del paisaje

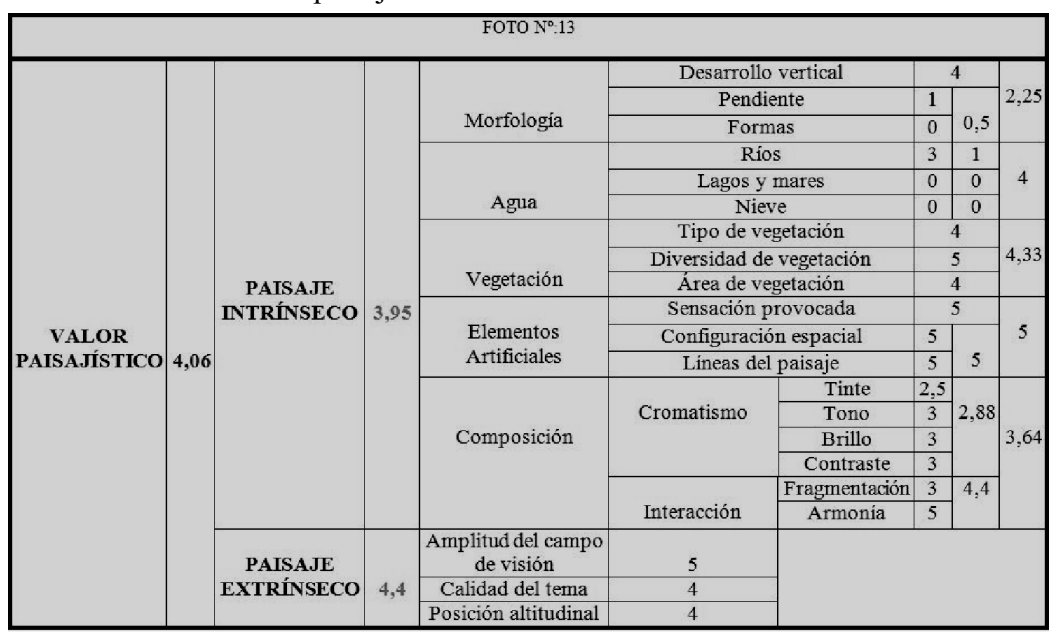

Fuente: Propia

De esta manera se obtiene una puntuación para cada fotografía. Dicho valor se compara con la media aritmética, obtenida de las votaciones realizadas por colaboradores anónimos en la página web del proyecto (Tabla 25). En lo que respecta a la participación en la encuesta, ésta es de 230 personas, aunque no todas las valoraciones han sido válidas, ya que se han encontrada valoraciones incomprensibles; éstas han sido rechazadas. En total se emplearon para realizar los correspondientes cálculos 217 encuestas. La pági- 
na web ha estado instalada en el espacio web citado con anterioridad durante 3 meses y para su difusión ha sido anunciada en redes sociales a través de Internet, en foros de diversas universidades, en tablones de anuncios de diversas instituciones, buscando así, la mayor participación de gente de diversa procedencia, edad y formación educativa.

\section{RESULTADOS}

Tras la valoración de las 33 fotografías por el método de valoración subjetiva y por el método indirecto propuesto, cuyos resultados se exponen en la Tabla 25 se ha procedido a representar los resultados (Fig. 6) para compararlos y observar si existe una cierta correlación en las valoraciones. En las Tablas y Figuras siguientes podemos observar el nivel de participación en función de las diversas categorías.

Tabla 22. Participación en función de la provincia

\begin{tabular}{|l|c|}
\hline \multicolumn{1}{|c|}{ Provincia } & Participación \\
\hline Galicia & $76,4 \%$ \\
\hline Madrid & $8,4 \%$ \\
\hline Asturias & $4,5 \%$ \\
\hline Andalucía & $4,5 \%$ \\
\hline Castilla-León & $1,7 \%$ \\
\hline Aragón & $1,1 \%$ \\
\hline Baleares & $0,6 \%$ \\
\hline Canarias & $0,6 \%$ \\
\hline Extremadura & $0,6 \%$ \\
\hline Murcia & $0,6 \%$ \\
\hline País Vasco & $0,6 \%$ \\
\hline Valencia & $0,6 \%$ \\
\hline
\end{tabular}

Fuente: Propia

Tabla 23. Participación en función del país

\begin{tabular}{|l|c|}
\hline \multicolumn{1}{|c|}{ País } & Participación \\
\hline España & $82 \%$ \\
\hline Vietnan & $6 \%$ \\
\hline Chile & $4 \%$ \\
\hline Colombia & $0,9 \%$ \\
\hline Inglaterra & $0,9 \%$ \\
\hline México & $0,9 \%$ \\
\hline Venezuela & $0,9 \%$ \\
\hline Alemania & $0,5 \%$ \\
\hline China & $0,5 \%$ \\
\hline E.E.U.U. & $0,5 \%$ \\
\hline Finlandia & $0,5 \%$ \\
\hline Francia & $0,5 \%$ \\
\hline Italia & $0,5 \%$ \\
\hline Madagascar & $0,5 \%$ \\
\hline Perú & $0,5 \%$ \\
\hline Polonia & $0,5 \%$ \\
\hline Portugal & $0,5 \%$ \\
\hline
\end{tabular}

Fuente: Propia 
Figura 4. Participación en función de la edad

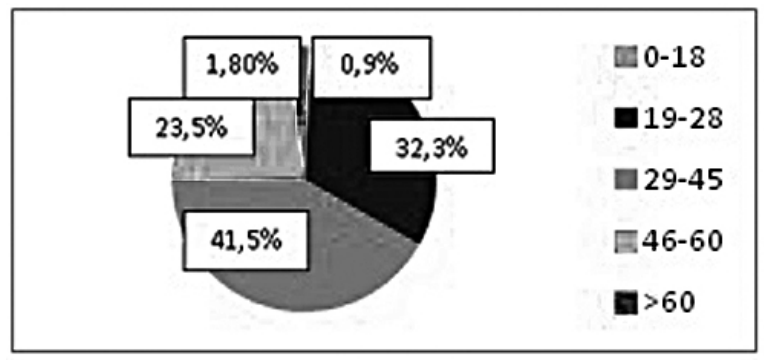

Fuente: Propia

Tabla 24. Participación en función de los estudios

\begin{tabular}{|l|c|}
\hline \multicolumn{1}{|c|}{ Nivel educativo } & Participación \\
\hline Doctores relacionados con M. Ambiente & $8,8 \%$ \\
\hline Doctores no relacionados con M. Ambiente & $5,5 \%$ \\
\hline Ing. Superiores relacionados con M. Ambiente & $22,6 \%$ \\
\hline Ing. Superiores no relacionados con M. Ambiente & $10,6 \%$ \\
\hline Ing. Técnicos relacionados con M. Ambiente & $2,8 \%$ \\
\hline Ing. Técnicos no relacionados con M. Ambiente & $1,4 \%$ \\
\hline Licenciados relacionados con M. Ambiente & $6,5 \%$ \\
\hline Licenciados no relacionados con M. Ambiente & $20,7 \%$ \\
\hline Diplomados relacionados con M. Ambiente & $1,8 \%$ \\
\hline Diplomados no relacionados con M. Ambiente & $6,5 \%$ \\
\hline Medios & $9,2 \%$ \\
\hline Básicos & $3,7 \%$ \\
\hline
\end{tabular}

Fuente: Propia. la valoración del paisaje 
Tabla 25. Comparación entre método directo e indirecto para

\begin{tabular}{|c|c|c|c|c|}
\hline$N^{0}$ de fotografía & Método directo & Método indirecto & Error & Desv. est. \\
\hline 1 & 6,80 & 5,08 & 1,72 & 1,99 \\
\hline 2 & 4,00 & 4,72 & $-0,72$ & 2,52 \\
\hline 3 & 8,56 & 7,05 & 1,51 & 1,61 \\
\hline 4 & 6,77 & 7,26 & $-0,49$ & 2,40 \\
\hline 5 & 3,05 & 2,96 & 0,09 & 2,76 \\
\hline 6 & 6,88 & 5,22 & 1,66 & 2,55 \\
\hline 7 & 8,41 & 7,92 & 0,49 & 1,84 \\
\hline 8 & 4,76 & 5,74 & $-0,98$ & 2,73 \\
\hline 9 & 4,73 & 5,44 & $-0,71$ & 2,55 \\
\hline 10 & 7,06 & 7,84 & $-0,78$ & 2,22 \\
\hline 11 & 3,19 & 5,04 & $-1,85$ & 2,42 \\
\hline 12 & 6,86 & 7,26 & $-0,40$ & 2,46 \\
\hline 13 & 7,95 & 8,12 & $-0,17$ & 1,81 \\
\hline 14 & 8,54 & 8,54 & 0,00 & 1,63 \\
\hline 15 & 7,41 & 5,58 & 1,83 & 2,22 \\
\hline 16 & 3,08 & 3,10 & $-0,02$ & 2,66 \\
\hline 17 & 7,71 & 7,68 & 0,03 & 1,84 \\
\hline 18 & 6,02 & 6,62 & $-0,60$ & 2,61 \\
\hline 19 & 8,40 & 8,30 & 0,10 & 1,80 \\
\hline 20 & 4,16 & 3,20 & 0,96 & 2,62 \\
\hline 21 & 8,72 & 8,16 & 0,56 & 1,72 \\
\hline 22 & 8,55 & 7,32 & 1,23 & 1,74 \\
\hline 23 & 4,96 & 4,50 & 0,46 & 2,52 \\
\hline 24 & 3,32 & 3,12 & 0,20 & 2,76 \\
\hline 25 & 3,75 & 4,98 & $-1,23$ & 2,40 \\
\hline 26 & 0,95 & 2,46 & $-1,51$ & 2,18 \\
\hline 27 & 2,93 & 5,44 & $-2,51$ & 2,44 \\
\hline 28 & 8,13 & 5,26 & 2,87 & 1,94 \\
\hline 29 & 2,10 & 2,30 & $-0,20$ & 2,57 \\
\hline 30 & 1,63 & 4,56 & $-2,93$ & 2,37 \\
\hline 31 & 7,12 & 5,92 & 1,20 & 1,90 \\
\hline 32 & 8,74 & 8,54 & 0,20 & 1,78 \\
\hline 33 & 8,96 & 8,96 & $-0,55$ & 1,45 \\
\hline Media & 5,88 & 5,90 & $-0,54$ & 2,21 \\
\hline Des. estándar & 2,44 & 1,98 & 1,25 & 0,39 \\
\hline
\end{tabular}

Fuente: Propia 
Figura 5. Participación de la población urbana y rural en la experiencia

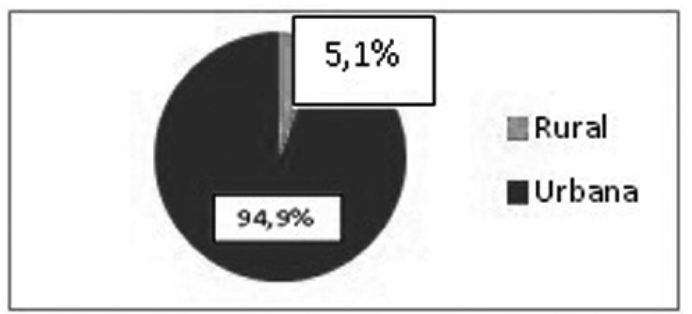

Fuente: Propia

Al representar gráficamente, en el eje de abscisas están indicados los resultados de la valoración realizada por el método indirecto, mientras que en el eje de ordenadas, se encuentran los resultados de la evaluación subjetiva o directa.

Figura 6. Correlaciones de los resultados

\section{INTERPRETACION ENCUESTA}

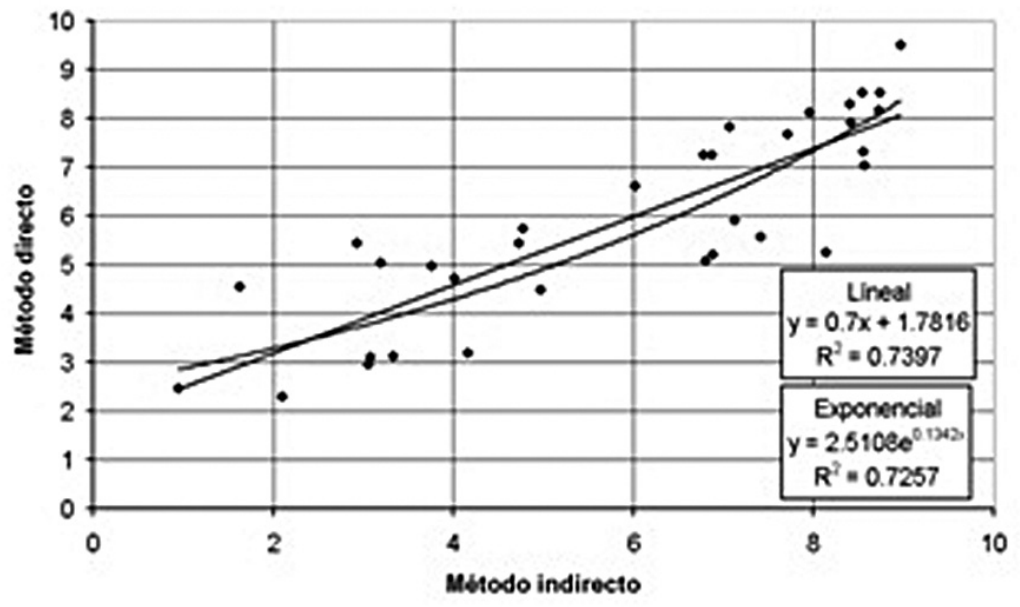

Fuente: Propia

Por lo tanto al observarse un R2 $=0,74$ se extrae la posibilidad de correlación lineal entre los dos métodos. Según Alejano et al. (2009) el método indirecto potencia los paisajes bonitos, lo cual queda reflejado en sus correlaciones, ya que obtiene una $\mathrm{R} 2=0,67$ para un ajuste lineal y un $\mathrm{R} 2=0,73$ para un ajuste exponencial. El hecho de que la anterior experiencia se haya llevado a cabo con 16 votaciones, realizadas las mayoría por estudiantes universitarios, hace que la subjetividad personal entre en juego, mientras que en este último estudio al tratar con 217 votaciones realizadas por 
personas de distintas procedencias, edades, ámbitos y estudios se consiguen eliminar, en gran parte, los factores sensitivos y perceptivos inherentes al observador.

\section{APLICACIÓN}

A continuación se presenta el estudio de restauración realizado para una cantera abandonada de piedra ornamental en el ayuntamiento de Marbella, Andalucía. Se trata de la cantera de Nagüeles y presenta la necesidad de una restauración debido a su gran valor paisajístico y medioambiental, ya que se encuentra en una zona LIC (Lugar de Importancia Comunitaria) y existe un alto grado de posibilidad de futuro empleo de la zona como Parque Natural, debido a la gran importancia que está obteniendo la Cueva de Nagüeles. Esta región se encuentra próxima a los núcleos urbanos de Marbella y San Pedro. Otra de las causas de esta posible restauración es el deterioro progresivo que se está provocando a causa del abandono del terreno y de los usos y vertidos incontrolados. También existe la asignación de fondos del Plan Cualifica (Plan de Recualificación Turística de la Costa del Sol) para la creación de un gran parque periurbano de 32 Has. en el entorno.

Figura 7. Fotografía actual de la cantera de Nagüeles (Málaga)

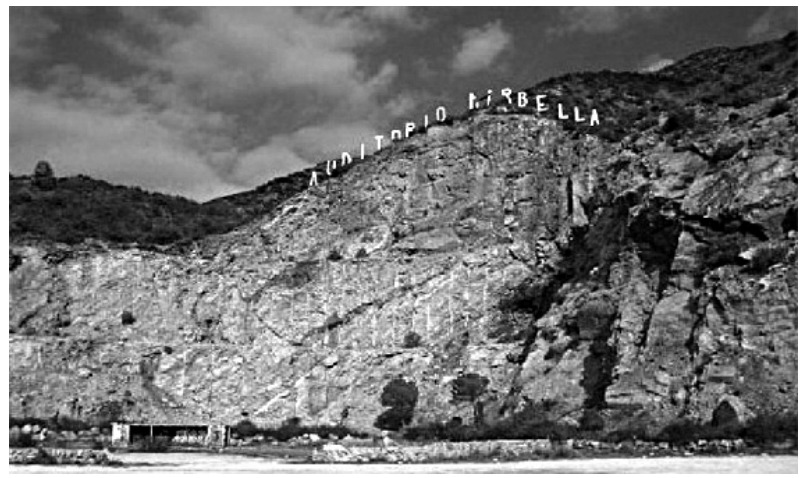

Fuente: Junta de Andalucía, 2007

Tras el empleo del método VPAI para la valoración de la instantánea presente en la Figura 7, se obtiene como resultado un 3,5. Es entonces cuando por métodos y programas informáticos se provoca la recreación virtual de la nueva imagen de la zona. Para ello en la simulación lo que se realiza son las siguientes pautas:

A. Eliminación de las letras del auditorio.

B. Soterramiento de decantadores en desuso.

C. Rellano del mirador en la parte alta del relleno principal a cota $282 \mathrm{~m}$.

D. Reforestación de taludes y zonas degradadas del pinar.

Con respecto a esta reforestación se llevará a cabo con pinos piñoneros (Pinus pinae). Pueden tener más de 25 metros de altura, con un tronco único, rectilíneo y vertical que se eleva sin ramificaciones en su primera mitad o más arriba, para después abrirse mediante ramas de similar grosor en una copa redondeada y achatada, en forma 
de sombrilla. La superficie del tronco se caracteriza por disponer de placas de color grisáceo, separadas por grietas rojizas. Prefiere los suelos arenosos, de ahí su elección.

Pinus pinea es natural de toda la franja mediterránea, siendo en la Península Ibérica más habitual en la zona centro y sur. Se desarrolla normalmente en un rango de alturas que van desde el nivel del mar hasta los 1000 ó 1200 metros sobre el nivel del mar, formando bosques monoespecíficos preferentemente en suelos silíceos. Es una especie heliófila, resiste muy bien la sequía estival y soporta heladas no muy extremas.

Una vez obtenida la simulación, establecida en la Figura 8, se realiza la valoración del "nuevo" paisaje mediante el VPAI. La valoración obtenida es de 6.6; esto nos indica que la restauración indicada y llevada a cabo mediante las aplicaciones informáticas correspondientes provoca un aumento de la calidad del paisaje. Esto se traduce en un aumento de la integración paisajística.

Figura 8. Recreación de la restauración

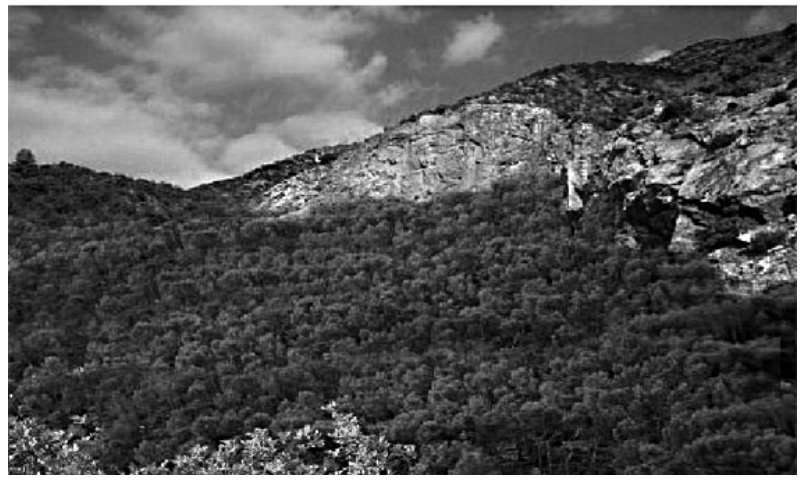

Fuente: Junta de Andalucía, 2007

\section{CONCLUSIONES}

Como queda demostrado, se ha cubierto el objetivo general de la experiencia, que era la elaboración de un método de valoración de paisaje, en el cual se eliminen razonablemente los factores de subjetividad a la hora de valorar un paisaje, obteniendo una opinión representativa de la sociedad, evitando así la interacción de las condiciones y mecanismos sensitivos y perceptivos inherentes al observador: forma de mirar, capacidad de imaginación, actitud en el momento de la contemplación, etc. que son diferentes para cada persona.

Se observa que existe una correlación razonable $(\mathrm{R} 2=0.74$ en el caso progresión lineal) a pesar de la enorme dispersión de la encuesta desarrollada, motivada tanto por preferencias personales como por los diferentes criterios de evaluación de los participantes. Las preferencias personales se ven claramente reflejadas en la Tabla 25, donde se observa como la mayoría de las imágenes tienen un amplio espectro de valoraciones.

La aplicación fundamental que se extrae de este método, es la posibilidad de valorar futuras restauraciones de escombreras, canteras, taludes, etc., simuladas por programas informáticos y descubrir el grado de integración paisajística en su entor- 
no. De esta manera se podrá escoger la mejor opción posible de acuerdo con los criterios de valoración del paisaje y así adoptar una solución de compromiso acorde con los objetivos y características del proyecto y su entorno, ya sean técnicos, económicos, sociales o ecológicos, entre otros.

La apuesta social por la conservación del medio ambiente y los recursos paisajísticos y el nuevo marco normativo creado tras la Convención Europea del Paisaje generan unas importantes perspectivas de futuro y una enorme oportunidad sobre este tipo de estudios que cada vez son más abundantes en estudios de impacto ambiental y en estudios de ordenación territorial y urbana.

Es importante tener en cuenta que este tipo de estudios pueden ayudar a reducir tensiones ante conflictos sociales provocados por el rechazo social hacia ciertas actuaciones transformadoras del paisaje. Un conocimiento por parte del personal relacionado con la minería y la ingeniería civil de estas técnicas de evaluación del paisaje, permitiría mejorar los resultados finales de la calidad escénica de los taludes con un bajo coste económico y con una dedicación pequeña de los proyectos, con respecto a dejar agua en la zona, aumentar la cobertura vegetal y favorecer la vegetación rupícola, aumentar la diversidad de especies a repoblar, elección de los materiales de recubrimiento y otras medidas sencillas con las que se puede mejorar la estética del talud.

El sólo hecho de tener en cuenta estos aspectos servirá para mejorar los resultados finales de los procesos de explotación.

\section{REFERENCIAS}

ALBERRUCHE, E. (2005). "Máster de aprovechamiento de los recursos minerales". Lima, perú.

ALBERRUCHE, E. (2002). El análisis de la fragilidad visual mediante Sistemas de Información Geográfica. En: Los sistemas de información geográficas en la gestión de los riesgos geológicos y el medio ambiente. 219-230.

ALEJANO, L.R., EIRAS J.A., ALBERRUCHE E. (2009). Aplicación de las técnicas de valoración del paisaje al ámbito de la ingeniería de taludes. En IngeoPress. 186: 58- 68.

ANDRADE PÉREZ et al. (1998). Zonificación ecológica y caracterización de las unidades ecológicas del paisaje en la cuenca hidrográfica del río Sinú. Colombia. 6-89.

B.L.M. (U.S.D.I. Bureau of Land Management). (1980). Visual resource management program. Div. of recreation and cultural resource. Government Printing Office. Washington DC, EEUU.

B.O.E., num 31, Martes 5 de febrero (2008).

CRAIK, K.H. (1975). Individual variations in landscapes description. En: Landscape assessment: values, perceptions and resources: 130-150. Dowden, Hurchinson \& Ross Inc., Stroudsburg, Pennsylvania, USA.

ERVIN H. ZUBE. (1987). Perceived land use patterns and landscape values. En: Landscape Ecology vol. 1 no. 1 pp 37-45. SPB Academic Publishing, The Hague. 
ITGE. (1995). Ordenación Minero-Ambiental del yacimiento de Pizarras Ornamentales de La Cabrera (León).Inédito. Servicio de Documentación del Instituto Geológico y Minero de España (IGME).

FORMAN R.T.T.; DEBLINGER R. (2000). The ecological road-effect zone of a Massachusetts (U.S.A.) suburban highway. En: Conservation Biology 14:36-46.

GÓMEZ OREA, D. (1978). El medio fisico y la planificación.

GONZÁLEZ BERNÁLDEZ, F. (1981).Ecología y paisaje. Blume. Barcelona.

HAINES-YOUNG, R.; GREEN, D.R.; COUSINS, S.H. (1993). Landscape Ecology and spatial information systems. En: Landscape Ecology and GIS. pp.3-8.

HIGUCHI, T. (1975). The Visual and Spatial Structure of Landscape. Gihodo Publishing. Tokyo.

LEI 7/2008 do 7 de xullo de Protección da paisaxe de Galicia (2008)

LENO CERRO, F. (1993). Técnicas de evaluación del potencial turístico. Madrid. Ministerio de Industria, Comercio y Turismo.

MARÍ COSTA, V. (2003). Ecohistoria del paisaje agrario: Una aplicación para el campo pituso. $\mathrm{Pp}$ 240-250.

MUÑOZ PEDREROS, A. et al. (1993). Evaluación del paisaje en un humedal del sur de Chile: el caso del río Valdivia (X Región). Revista Chilena de Historia Natural 66: 403-118.

MUÑOZ PEDREROS, A. et al. (2000). Variabilidad de la percepción del recurso paisaje en el sur de Chile. Revista Chilena de Historia Natural 73: 729-738.

MUÑOZ PEDREROS, A. (2004). La evaluación del paisaje: una herramienta de gestión ambiental. En Revista Chilena de Historia Natural 77. 139-156.

NAVARRO J.; JONTE M. (1996). La erosión hídrica en los desmontes de la N-610 y N-611 en la circunvalación de la ciudad de Palencia. Rutas. 54:35-42.

PAQUET, J.; BÉLANGER L. (1997). Public acceptability thresholds of clearcutting to maintain visual quality of boreal balsam fir landscapes. En: Forest Science 43: 46-55

TORMO, J.; BOCHET, E.; GARCÍA-FAYOS. (2009). Restauración y revegetación de taludes de carreteras en ambientes mediterráneos semiáridos: procesos edáficos determinantes para el éxito. Ecosistemas: revista científica y técnica de ciencia y medio ambiente.

TROLL C. (1989). Landscape ecology (geoecology) and biogeocenology-a terminology study. Geoforum 8/57:43-46.

VILLARINO, M. T. (1985). El paisaje. En: Curso sobre evaluaciones de impacto ambiental. Dirección General del Medio Ambiente. M.O.P.U.,Madrid.

VILlOTA, H. (1992). El Sistema CIAF de Clasificación Fisiográfica del Terreno. En: Revista CIAF. Vol. 13(1): 55 - 70.

UNESCO. (1972). Convention Concerning the Protection of World Cultural and Natural Heritage. www.whc.unesco.org/en/conventiontext/

U.S.D.A. FOREST SERVICE. (1974). The visual management system. National forest landscape management. vol.2. Chapter 1. Forest Service. U.S.D.A. Government Printing Office.

ZONNEVELD, I.S. (1995). Land ecology. SPB Academic Publishing, Amsterdam. 